\title{
Método mãe canguru: uma análise supletiva sobre aassistência de enfermagem
}

\author{
Kangaroo mother method: a supplemental analysis on nursing care \\ Método madre canguro: un análisis supletivo sobre el cuidado de enfermería
}

Recebido: 10/08/2021 | Revisado: 14/08/2021 | Aceito: 15/08/2021 | Publicado: 17/08/2021

\author{
Maria Valquíria Alves dos Santos \\ ORCID: https://orcid.org/0000-0003-2440-7262 \\ Centro Universitário Estácio de Sergipe, Brasil \\ E-mail: valquiria.al16@gmail.com \\ Ana Fátima Souza Melo de Andrade \\ ORCID: https://orcid.org/0000-0002-7024-6175 \\ Centro Universitário Estácio de Sergipe, Brasil \\ E-mail: anafatimamelo@hotmail.com \\ Weber de Santana Teles \\ ORCID: https://orcid.org/0000-0003-1770-8278 \\ Centro de Hemoterapia de Sergipe, Brasil \\ E-mail: arteecura@hotmail.com \\ Max Cruz da Silva \\ ORCID: https://orcid.org/0000-0002-6944-5986 \\ Faculdade Pio Décimo, Brasil \\ E-mail: maxlfi@hotmail.com \\ Ruth Cristini Torres \\ ORCID: https://orcid.org/0000-0002-8664-192X \\ Instituto de Hematologia e Hemoterapia de Sergipe, Brasil \\ E-mail: ruthcristini@gmail.com \\ Maria Hozana Santos Silva \\ ORCID: https://orcid.org/0000-0001-5742-5366 \\ Faculdade Ages de Medicina, Brasil \\ E-mail: hosana_p@hotmail.com \\ Marcel Vinícius Cunha Azevedo \\ ORCID: https://orcid.org/0000-0002-5312-3333 \\ Centro Universitário Estácio Sergipe, Brasil \\ E-mail: marcelvinicius49@gmail.com \\ Taíssa Alice Soledade Calasans \\ ORCID: https://orcid.org/0000-0003-0460-4437 \\ Universidade Tiradentes, Brasil \\ E-mail: taissa.asc@gmail.com \\ Paulo Celso Curvelo Santos Junior \\ ORCID: https://orcid.org/0000-0001-5834-6782 \\ Universidade Tiradentes, Brasil \\ E-mail: paulo.curvelo.jr@gmail.com \\ Ângela Maria Melo Sá Barros \\ ORCID: https://orcid.org/0000-0003-4087-3247 \\ Universidade Federal do Rio de Janeiro, Brasil \\ E-mail: angelsamelo@hotmail.com \\ Alejandra Debbo \\ ORCID: https://orcid.org/0000-0002-7743-5921 \\ Universidade Tiradentes, Brasil \\ E-mail: aledebbo@hotmail.com
}

\begin{abstract}
Resumo
O objetivo do presente estudo foi de identificar na literatura os benefícios do método mãe canguru e atribuições do enfermeiro quanto ao método, discorrer sobre o assunto e caracterizar sobre seus respectivos benefícios. O método realizado na pesquisa foi de revisão bibliográfica de natureza qualitativa, estudo retrospectivo, exploratório e descritivo, utilizando as bases de dados indexada, a saber, Literatura Latino-Americana e do Caribe em Ciências da Saúde (LILACS), Brasil Scientific Electronic Library Online (SCIELO), Sistema Online de Busca e Análise de Literatura Médica (Medline) e Google Acadêmico. O este método é utilizado como proposta de humanização não relacionando somente ao nascimento e ao parto, mas à estadia do recém-nascido de baixo peso, com necessidade de hospitalização,-e às situações que dificultam a relação do binômio mãe e filho. Além de ser um gesto carinhoso,ométodo estabelece maior apego e segurança ao resgatar o contato íntimo entre mãe e recém-nascido e, por sua vez, nos mostra que não é uma substituição de tecnologia, e sim uma abordagem mais ampla, onde o método consiste numa maneira de cuidado, pautada na atitude da equipe de enfermagem diante do recém-nascido e sua família, a partir de um contexto que não se limita ao conhecimento técnico específico. Para o alcance dessa finalidade, é importante a atuação do profissional de
\end{abstract}


enfermagem na orientação, no preparo e no acolhimento da mãe e da família, além da imprescindível participação da rede de apoio familiar.

Palavras-chave: Cuidados de enfermagem; Método canguru; Recém-nascido.

\begin{abstract}
The aim of the present study was to identify in the literature the benefits of the kangaroo mother method and nurses' attributions regarding the method, to discuss the subject and characterize its respective benefits. The method used in the research was a bibliographic review of a qualitative nature, a retrospective, exploratory and descriptive study, using the indexed databases, namely, Latin American and Caribbean Literature on Health Sciences (LILACS), Brazil Scientific Electronic Library Online (SCIELO), Online Medical Literature Search and Analysis System (Medline) and Academic Google. This method is used as a proposal for humanization, not only relating to birth and delivery, but to the stay of low-weight newborns, with the need for hospitalization, and to situations that hinder the relationship between mother and child. In addition to being a loving gesture, the method establishes greater attachment and security by rescuing the intimate contact between mother and newborn and, in turn, shows us that it is not a replacement for technology, but a broader approach, where the method consists of a way of care, based on the attitude of the nursing team towards the newborn and his family, from a context that is not limited to specific technical knowledge. To achieve this purpose, it is important for the nursing professional to provide guidance, preparation and care for the mother and family, in addition to the essential participation of the family support network.
\end{abstract}

Keywords: Nursing care; Kangaroo method; Newborn.

\title{
Resumen
}

El objetivo del presente estudio fue identificar en la literatura los beneficios del método madre canguro y las atribuciones de las enfermeras respecto al método, discutir el tema y caracterizar sus respectivos beneficios. El método utilizado en la investigación fue una revisión bibliográfica de carácter cualitativo, un estudio retrospectivo, exploratorio y descriptivo, utilizando las bases de datos indexadas, a saber, Literatura Latinoamericana y del Caribe en Ciencias de la Salud (LILACS), Biblioteca Científica Electrónica de Brasil en Línea (SCIELO), Sistema de análisis y búsqueda de literatura médica en línea (Medline) y Google académico. Este método se utiliza como una propuesta de humanización, no solo en relación con el parto y parto, sino con la estancia de recién nacidos de bajo peso, con necesidad de hospitalización y con situaciones que dificultan la relación madre-hijo. Además de ser un gesto amoroso, el método establece un mayor apego y seguridad al rescatar el contacto íntimo entre madre y recién nacido y, a su vez, nos muestra que no es un sustituto de la tecnología, sino un enfoque más amplio, donde el método consiste en una forma de cuidado, basada en la actitud del equipo de enfermería hacia el recién nacido y su familia, desde un contexto que no se limita a conocimientos técnicos específicos. Para lograr este propósito, es importante que el profesional de enfermería brinde orientación, preparación y cuidado a la madre y la familia, además de la participación esencial de la red de apoyo familiar.

Palabras clave: Atención de enfermería; Método canguro; Recién nacido.

\section{Introdução}

O método mãe canguru foi idealizado na Colômbia, em 1979, se constituindo em uma alteração do paradigma da atenção perinatal e uma associação entre a humanização e os avanços tecnológicos. Sua ação consiste em manter o recémnascido de baixo peso, em contato pele a pele, na posição vertical junto ao tóraxda mãe, pai ou outro familiar, de maneira segura e acompanhada de suporte assistencial de uma equipe de enfermagem treinada (Costa, et al., 2012).

O acréscimo da incidência da prematuridade e do baixo peso ao nascer em capitais e cidades de densa população no Brasil aponta o nascimento pré-termo como um dos fatores determinantes para a mortalidade neonatal. No ano de 2008, no Brasil, $6,7 \%$ dos nascidos vivos foram pré-termo com uma variação entre 4,8\% e 7,7\%, nas regiões Norte e Sudeste, sendo que esse aumento se relaciona com as taxascrescentes de cesarianas programadas com interrupção indevida da gravidez, sem justificativa obstétrica, aumentando assim o perigo risco de morte infantil e perinatal (Almeida, et al., 2010).

Transformações tecnológicas reprodutivas que possibilitam a fecundação ocasionaram um impacto relevante para a saúde materna e neonatal, pois o progresso tecnológico permitiu o aperfeiçoamento das técnicas de reprodução assistida, aumentando as chances do desenvolvimento da gestação e o aparecimento de adversidades e importantes conquistas. Porém, é notório que as técnicas de reprodução assistida influem nos resultados neonatais, por proporcionaram a essas gestações um elevado índicede nascimentos prematuros, o que aumenta as complicações neonatais, além da doença hipertensiva específica da 
gravidez que resulta, muitas vezes, em nascimento de recém-nascidos prematuros, restrição de crescimento intrauterinoe a sepse (Araújo, et al., 2010).

O nascimento de um recém-nascido prematuro pode representar um instante de crise para a família e resultar em desequilíbrio familiar, com reflexo na incapacidade temporária da mãe e do pai em tomarem decisão ou responderem adequadamente às demandas, com esta situação provocando medo, tristeza e constrangimento (Arivabene \& Tyrrell, 2010).

$\mathrm{O}$ número elevado de nascimentos prematuros e de baixo peso e a necessidade de suporte aos profissionais de enfermagem conduziram o Ministérioda Saúde a apoiar e padronizar ações nas unidades neonatais instituindo em 05/07/2000, a Norma de Atenção Humanizada ao Recém-Nascido de Baixo Peso (autor, ano).Muito além desse preceito está a importância que o método mãe canguru possui no intuito de contribuir para modificações na atitude profissional voltada àhumanização da assistência ao recém-nascido (Casati, et al., 2010).

Para sua efetividade, é preciso que os profissionais de enfermagem que atuam com esta ferramenta adquiram conhecimento dos procedimentos, propósitos e atos assistenciais e de cuidados que abrangem esse método incluindo, o preparo precoce da genitora para o cuidado com recém-nascido; o favorecimento do contato entre a mãe e o recém-nascido; a contribuição para aumentar o vínculo afetivo entre o recém-nascido e a genitora; a promoção da estabilidade térmica e demais cuidados para o desenvolvimento do recém-nascido (Conz, et al., 2009).

Os benefícios do método mãe canguru abrangem mais do que o aumento do vínculo, menor tempo de separação entre mãe e recém-nascido, o que evita longos períodos sem estimulação sensorial; contribui no estímulo ao aleitamentomaterno, o que favorece a precocidade e duração da mamada; maior segurança,confiança e competência da mãe e/ou do pai no cuidado do recém-nascido mesmo depois da alta hospitalar; melhor controle térmico; menor número de recémnascido em unidades de cuidados intermediários por causa da maior rotatividadede leitos; melhor relacionamento da família com a equipe de saúde; redução da infecção hospitalar e menor permanência hospitalar (Henning, et al., 2010).

A Organização Mundial da Saúde (OMS) considera prematuro, o nascimento anterior a trinta e sete semanas gestacionais, incluindo os recém- nascidos com peso inferior a um quilo e meio, e os com peso inferior a um quilo (Machado, et al., 2012).

A adaptação desses recém-nascidos acontece em uma Unidade de Terapia Intensiva Neonatal (UTIN), a qual precisa oferecer um suporte tecnológicoe uma equipe de saúde preparada para o recebimento do recém-nascido (Lélis,et al., 2009).

Os progressos cuidados neonatais tem demonstrado um aumento na sobrevivência dos recém-nascidos, no entanto existe a exigência de um atendimento diferenciado, pois esses apresentam maior vulnerabilidade quanto àsmodificações no seu desenvolvimento, podendo significar uma sequela permanente (Santana, et al., 2013).

Assim, compete aos profissionais de enfermagem uma atuação criteriosa e mais flexível, associada ao preparo técnicocientífico adequado, convergindo para a humanização e a qualidade da assistência aos recém-nascidos e suas famílias(Arivabene \& Tyrrell, 2010).

O vínculo entre o recém-nascido e a mãe fica difícil quando ocorre um parto pré-termo, em virtude de o recém-nascido permanecer por muito tempo em uma UTI, porém essa situação pode ser minorada com o auxílio de uma equipe multiprofissional bem preparada, onde se insere o profissional de enfermagem quemantém contato mais prolongado com a mãe e a família, escutando de maneira sensível seguido de orientação adequada a cada situação, facilitando a interação da mãe, pai e família com o recém-nascido dentre outras questões relativas à permanência da mãe e do recém-nascido. (Machado, et al., 2012).

O método mãe canguru teve início em 1997, no Brasil, mais precisamente InstitutoMaterno-Infantil de Pernambuco (IMIP), com a implantação da enfermaria canguru. Vale ressaltar que este estava sendo adotado pelo Hospital Estadual Guilherme Álvaro, desde 1991, na cidade de Santos-SP (Neves, et al., 2010). 
Este método foi ampliado a partir de sua inserção, em 1999, da Política de Atenção Humanizada ao Recém-Nascido de Baixo Peso - Método Canguru, servindo como mediador na Área Técnica de Saúde da Criança e Aleitamento Materno do Ministério da Saúde, integrando as ações de humanização da assistência à criança, à família e aos cuidadores, respeitando suas característicase individualidades (Maia, et al., 2011).

Esse método possibilita o resgate do tempo interrompido da mãe do recém-nascido na eventualidade do nascimento prétermo, construindo assim um vínculo a partir do momento em que a mãe coloca o recém-nascido junto ao seu corpo, gerando espaço para uma reconstrução rítmica (Lélis, et al., 2009).

O Ministério da Saúde (ano) considera que a utilização do método mçae canguru precisa iniciar de maneira crescente e precoce, envolvendo o contato pele a pele que aos poucos evolui até a posição canguru, por livre escolha da família, pelo tempo considerado suficiente,permitindo a participação da mãe, do pai e da família nos cuidados neonatais (Matos \& Campos, 2009).

As atitudes primordiais para a implantação são de acolher a mãe, o pai e os familiares na unidade neonatal; não sobrecarregando com muitas informações; esclarecendo sobre as condições de saúde do recém-nascido, e sobre os cuidados a ele dispensados, a equipe, as rotinas e o funcionamento da UTI neonatal; proporcionando a aproximação progressiva, reduzindo as dificuldades para a realização do toque, dos cuidados e do contato pele a pele (Moreira, et al., 2009).

Na primeira fase da utilização do método mãe canguru é indispensável que o profissional de enfermagem oriente sobre a amamentação e a ordenha de leite, incentivando aparticipação do pai em todos os processos desenvolvidos; ajudar os pais no entendimento das características do recém-nascido; posicionamento apropriadodo recém-nascido e medidas contra dor, proporcionando mais conforto e melhor padrão de sono; realizar pesagem, higiene, aspiração de tubo orotraqueal e punções, procurando o bem-estar do recém-nascido; proporcionar cadeira própriapara o repouso da mãe (Moreira, et al., 2009).

$\mathrm{Na}$ segunda fase é preciso que aconteça a estabilidade clínica do recém- nascido, que o mesmo esteja com nutrição enteral plena e peso mínimo de $1.250 \mathrm{~g}$. Dentre as recomendações importantes que o profissional de enfermagem precisa transmitir nessa fase está o tempo ilimitado na posição canguru; auxiliar para quea mãe se sinta segura tanto no posicionamento do recémnascido quanto na identificação de sinais de alerta; disponibilizar berço, com possibilidade de elevação da cabeceira; estimular a participação do pai; não obrigar a permanênciada mãe na unidade no decorrer da internação do recém-nascido, permitindo que ela fique ausente do hospital, com direito a regressar quando possível; permitir acesso dos irmãos e avós; apoiar para o aleitamento materno; desenvolver atividades educativas para preparar a mãe com os cuidados do recém-nascido no domicílio (Queiroz \& Maranhão, 2012).

O profissional de enfermagem precisa avaliar as necessidades de cada puérpera antes da segunda fase, porque algumas mães não estão preparadas, ou não possuem disponibilidade para ficar no hospital. Sendo assim, o recém-nascidoprecisará receber cuidados tradicionais enquanto a família se organiza. Porém, independentemente do tempo de permanência, a presença da mãe tem que serem tempo integral nos dias que precedem a alta hospitalar (Sá, et al., 2010).

A alta da segunda fase está vinculada à alta hospitalar, porém no método mãe canguru precisará haver continuidade em casa, com acompanhamento ambulatorial. A decisão da alta precisará ser pensada desde que a mãe esteja psicologicamente motivada, com a obrigação materna e familiar para a realização da posição canguru pelo maior tempo possível, peso mínimo de $1.600 \mathrm{~g}$, sucção exclusiva aopeito ou, mãe e família habilitadas para realizar a complementação (Schmidt, etal., 2011).

A terceira fase se inicia com a alta hospitalar, sendo preciso o acompanhamento do recém-nascido pela equipe que o assistiu no decorrer da internação até que atinja o peso de $2.500 \mathrm{~g}$. Essa etapa não pode ficar limitada à presença de um ambulatório de seguimento, pois pode ser feita em qualquer unidade básica de saúde ou no domicílio, pelas equipes da Estratégia de Saúdeda Família (ESF) (Silva, et al., 2011).

A posição canguru necessita ser usada pelo maior tempo possível, então, se os pais quiserem podem escolher outra pessoa da família para auxiliar. Valeressaltar que muitas das atividades do dia-a-dia podem ser realizadas com o recém-nascido 
em posição canguru. As recomendações importantes para a terceira fase do método inclui o acompanhamento ambulatorial até que a criança alcance o peso de $2.500 \mathrm{~g}$; sugerir o primeiro retorno até 72 horas após a alta e os demais pelo menos uma vez por semana (Silva, et al., 2013).

A ausência do marido ou da família exige que o profissional de enfermagem ache maneiras de auxiliar nesta fase. A percepção do profissional de enfermagem a respeito do nível de embaraço da situação em que se encontra a mãe é primordial para detectar a necessidade de se acionar uma rede de apoio que acompanhe o recém-nascido durante a internação e depois da alta hospitalar (Veras \& Traverso-Yépez, 2011).

As indicações deste método são importantes para que se usufrua dos benefícios do mesmo da melhor maneira possível, sendo primordial que a sua realização e de todas as suas fases sejam realizadas corretamente e com orientação adequada (Arivabene \& Tyrrell, 2010).

Este método se objetiva quando é destinado a promover a alta precoce para os recém-nascidos, devido aos problemas das redes públicas de saúde que se enfrenta como a escassez de recursos tecnológicos, tendo a falta de incubadoras como um deles, sendo inevitável o aparecimento de infecções cruzadas, o desmame precoce pelo não incentivo ao aleitamento materno, e a preocupação maior das altas taxas de mortalidade neonatal (Schmidt, et al., 2011).

As recomendações para a implantação do método mãe canguru apontam os benefícios, principalmente para os países em desenvolvimento, pois é uma tecnologia praticável e apropriada para hospitais com recursos limitados, sendo um método alternativo ao excesso de tecnologia de ponta, sem desmerecê-la, pois, o métodoé de baixo custo (Conz, et al., 2009).

Relacionado as contra-indicações do método, ocorrem principalmente em relação à estabilidade clínica do bebê, que muitas vezes não pode ser colocado em posição canguru. Em outras ocasiões, o bebê necessita de suporte intensivo, ou seja, ventilação mecânica, incubadora, por causa do baixo peso, e isto também dificulta o uso do tratamento a partir do método mãe canguru (Arivabene \& Tyrrell, 2010).

A depender da gravidade de alguns recém-nascidos é dificultada a posição canguru, porque muitos destes se encontram intubados, sob ventilação mecânica impossibilitando assim, se torna inviável o uso por causa da instabilidade hemodinâmica desses recem-náscidos (Lélis, et al., 2009).

A assistência neonatal está passando por muitas modificações, sendo queo progresso nos cuidados intensivos ao recémnascido tem assegurado maior sobrevida, principalmente aos que estão em circunstância de prematuridade extrema (Sá, et al., 2010).

É notória a necessidade da associação entre tecnologia e a humanização do cuidado, sendo primordial a atuação dos profissionais de enfermagem neste tratamento, pelo maior contato com a mãe, pai e outros familiares. Dentre as ações do profissional de enfermagem estão as orientações específicas a respeito da utilização dos equipamentos que o recém-nascido irá precisar, sanando-lhes dúvidas e estimulando o contato precoce, além do incentivo à ordenha de leite para os recém-nascidos que ainda não podem sugar o seio materno e aleitamento materno para os que estiverem estáveis e com idade gestacional apropriada (Veras \& Traverso-Yépez, 2011).

Sendo assim, muitas alterações estão acontecendo no ambiente das unidades neonatais, de certa maneira acompanhando a tendência mundial, com situações propícias de contado imediato entre mãe e pai do recém-nascido prematuro, apesar de esse processo ser gradual, e consumir mais tempo além dos primeiros dias ou semanas do período pós-natal (Costa, et al., 2012).

A percepção distinta pelos profissionais de enfermagem que cuidam do recém-nascido é essencial perante a possibilidade de agir em conjunto à equipe de saúde para que reduzam o estresse e a dor, por intermédio do cuidado humanizado e individualizado (Almeida, et al., 2010). 
Os cuidados do profissional de enfermagem precisam abranger atos e planejamentos, tais como, analgesia não farmacológica e farmacológica para diminuir a dor. Além desse aspecto, seja realizável a possibilidade de reduzir ou impedir os efeitos nocivos para o desenvolvimento do recém-nascido, o que ajudará para uma recuperação mais veloz e uma assistência habilitada (Araújo, et al., 2010).

Cabe ao profissional de enfermagem manter uma relação de confiança com a mãe e a família do recém-nascido, informando e orientando quanto aos cuidados com o recém-nascido, de maneira objetiva e com linguagemsimples, facilitando a captação das orientações pela mãe. A interação entre os profissionais de enfermagem e as mães facilita a permanência no tratamento com o método mãe canguru, pois elas se sentirão autoconfiantes para cuidar do recém-nascido nos momentos mais frágeis, compartilhando seus temores, dúvidas e aflições no decorrer da internação do recém-nascido (Sá, et al., 2010).

Dentre as ações desenvolvidas pelo profissional de enfermagem na UTIN está a organização do ambiente, o planejamento e a execução dos cuidados, conforme a necessidade e a resposta de cada recém-nascido, contribuindo para uma assistência completa, de excelência e humanizada (Arivabene \& Tyrrell, 2010).

A capacitação dos profissionais de enfermagem para tomar conhecimento das necessidades de cada recém-nascido é muito importante, no sentido de que as condutas de rotina, na maioria das vezes, dolorosas e invasivas sejam feitas de maneira individualizada e singular, sendo que uma das primeiras atitudes é a observação esmerada das respostas comportamentais e fisiológicas do recém- nascido, procurando reduzir progressivamente e individualmente, o estresse e a dor, concorrendo para o conforto e segurança (Casati, et al., 2010).

Para que o cuidado do profissional de enfermagem ao recém-nascido seja considerado é importante abranger o atendimento às necessidades de repouso,calor, nutrição, higiene, adicionado da verificação e do atendimento contínuo (Conz, et al., 2009).

É de suma importância que o profissional de enfermagemsaiba identificar quando é possível adotar o o tratamento com o método mãe canguru em uma UTIN, ele precisa de ter experiência, vivência, e qualificações especiais com conhecimentos expressivosque proporcione o desenvolvimento de práticas fundamentadas técnica e cientificamente, assegurando a qualidade de assistência ao recém-nascido, aospais e sua família. Além disso, é primordial que o profissional de enfermagem conheça as recomendações e legislações vigentes para atuar em UTI, além de ser uma exigência a especialização em UTI (Arivabene \& Tyrrell, 2010).

Para adotar o uso deste método em UTIN é imprescindível que toda a equipe interdisciplinar possua conhecimentos básicos e critérios mínimos para que aconteça o sucesso, porém é importante que todos os profissionais estejam envolvidos e desejem sua implementação (Conz, et al., 2009).

O profissional de enfermagem precisa estar ciente que o método mãe canguru pode ser uma opção para a substituição da incubadora, mas não é um substituto da UTIN, nem da utilização de incubadoras radicalmente, porque a utilização das incubadoras possui as suas indicações estabelecidas. Este fato pode representar um conhecimento relativo pelo profissional de enfermagem sem o aprofundamento sobre o mesmo, podendo se tornar um fator dificultador para a adoção do mesmo na UTIN (Arivabene \& Tyrrell, 2010).

O objetivo do presente estudo foi de identificar na literatura os benefícios do método mãe canguru e atribuições do enfermeiro quanto ao método, discorrer sobre o assunto e caracterizar sobre os benefícios deste método.

\section{Metodologia}

Trata-se de uma pesquisa bibliográfica de natureza qualitativa. Foi realizado um estudo retrospectivo, exploratório e descritivo, utilizando as bases de dados indexada, a saber, Literatura Latino-Americana e do Caribe em Ciências da Saúde 
(LILACS), Brasil Scientific Electronic Library Online (SCIELO), Sistema Online de Busca e Análise de Literatura Médica (Medline) e Google Acadêmico.

Quanto aos aspectos éticos e legais, como não se trata de uma pesquisa envolvendo seres humanos, não houve necessidade no seguimento da resolução 466/12 do Conselho Nacional de Saúde.

Os descritores utilizados para selecionar os materiais foram: Cuidados de enfermagem, Método Canguru e RecémNascido. Foram utilizados o seguintes operadores boleanos: "and" e "or". As publicações foram selecionadas e realizou-se uma leitura, separando os materiais por similaridades.

Para coleta e análise dos dados que interessavam ao trabalho se utilizou um roteiro com os seguintes elementos: Título, ano de publicação, fonte e descritores.

Os critérios de inclusão para a definição dos artigos científicos que fizeram parte desta pesquisa foram: Artigos que abordassem o tema dos benefícios do método mãe canguru e a atuação do enfermeiro.

Os critérios de exclusão foram: Artigos que não tratassem do tema dosbenefícios do método mãe canguru e a atuação do enfermeiro e artigos que não respondessem aos critérios estabelecidos a partir dos objetivos do presente estudo.

\section{Resultados e Discussão}

Após a análise da literatura selecionada nas bases de dados citadas, fo identificado que o método mãe canguru vem como proposta de humanização não se dirigindo somente ao nascimento e ao parto, mas à estadia do recém-nascido baixo peso, com necessidade de hospitalização, no hospital e às situações que dificultam a relação do binômio mãe e filho (Costa, et al., 2012).

A humanização e a assistência neonatal implicam no contato precoce pele a pele da mãe com o recém-nascido, não sendo exclusividade das mães, podendo os pais participarem do programa. Essa prática humanizada aumenta muito o vínculo familiar, deixa o recém-nascido mais seguro e proporciona maior confiança aos pais no manuseio do recém-nascido, o que constitui uma relação importante parao desenvolvimento completo do recém-nascido (Silva, et al., 2020).

Além de ser um gesto carinhoso, este método estabelece maior apego e segurança ao resgatar o contato íntimo entre mãe e recém-nascido e, por sua vez, nos mostra que não é uma substituição de tecnologia, e sim uma abordagem mais ampla, onde o método consiste numa maneira de cuidado, pautada na atitude da equipe de enfermagem diante do recém-nascido e sua família, a partir de um contexto que não se limita ao conhecimento técnico específico (Ferreira, et al., 2012).

O cuidado individualizado tem elementos que impõem modificações no ambiente para reduzir os níveis de som e luz, organização de estratégias específicas para facilitar a auto-organização do bebê e o envolvimento dos pais, a partir de um plano individual conforme as respostas da criança aos procedimentos (Schmidt, et al., 2011).

A equipe de enfermagem tem o papel de apoiar e auxiliar os cuidados gerais, porém, são os pais que tem o papel principal na evolução do crescimento do recém-nascido. É importante que a mãe mantenha alguns critérios para não causar irritação no bebê como, por exemplo, não usar perfumes, cremes hidratantes com cheiro excessivo, colares, sutiã que possa machucar o bebê, etc (Almeida, et al., 2010).

O horário noturno requer alguns cuidados especiais tais como a posição que a mãe fica semisentada apoiada por travesseiros e almofadas mantendo a criança contra o peito em posição vertical evitando assim a apneia que é comumao recémnascido (Autor, ano).

Porém não basta aos profissionais de saúde conhecer, estarem disponíveise aceitarem a realização do método canguru, deve haver uma mudança de comportamento da equipe e da filosofia da instituição no sentido de buscar a humanização de assistência ao recém-nascidos sua família (Moreira, et al.,2009). 
Logo, os estudos demonstraram que esse método possibilita o resgate do tempo interrompido da mãe do recémnascido na eventualidade do nascimento pré-termo, construindo assim um vínculo a partir do momento em que a mãe coloca o recém-nascido junto ao seu corpo, gerando espaço para uma reconstrução rítmica.

\section{Considerações Finais}

A assistência e cuidados neonatais associados ao método mãe canguru reforçam seu valorpara a sobrevida e redução da morbidade de neonatos. Ao lado disso, a análisedas questões individuais que possam atender às necessidades de cada mãe, alémde significante, são informações essenciais para sua autodeterminação e independência, determinantes para o atingimento da cidadania.

O preparo da mãe para o cuidado do recém-nascido na volta ao lar é algoque precisa ser trabalhado desde a primeira fase deste tratamento, pois essa mãe não contará com o auxílio dos profissionais de enfermagem em seu cotidiano a partir do momento em que retornará para p o seu lar, necessitando desse prévio aprendizado para adquirir experiência e autonomia para realizar os cuidados.

Para o alcance dessa finalidade, é importante a atuação do profissional de enfermagem na orientação, no preparo e no acolhimento da mãe e da família, além da imprescindível participação da rede de apoio familiar.

Fica evidente a função essencial do profissional de enfermagem no atentimento aos recém-nascidos que utilizam o método,ao proporcionar um ambiente acolhedor, buscando entender cada necessidade, com os corretos encaminhamentos para possível solução e bem-estar da mulhere do recém-nascido.

Sendo assim, espera-se que este estudo sirva como modelo para o desenvolvimento de novas pesquisas que possibilitem a elaboração e implantação de estratégias que atendam aos propósitos do método mãe canguru e diminuam a insatisfação e angústia das mães.

\section{Referências}

Almeida, H., Venâncio, S. I., Sanches, M. T. C. \& Onuki, D. (2010). Impacto do método canguru nas taxas de aleitamento materno exclusivo em recém-nascidosde baixo peso. Jornal de Pediatria. 86(3), 250-3.

Araújo, C. L., Rios, C. T. F., Santos, M. H. \& Gonçalves, A. P. F (2010). Método mãe canguru: uma investigação da prática domiciliar. Revista Ciência \& Saúde Coletiva, 15(1), 301-7.

Arivabene, J. C. \& Tyrrell, M. A. R. (2010). Método mãe canguru: vivências maternas e contribuições para a enfermagem. Revista Latino- Americana de Enfermagem, 18(2), 262-8.

Casati, P. S., Oliveira, C. S. \& Paula, S. (2010). Método mãe canguru e suasassociações nos benefícios dos recém-nascidos baixo peso. UNICiências, CuiabáMT. 14(1), 135-46.

Conz, C. A., Merigji, M. A. B. \& Jesus, M. C. P. (2009). Promoção de vínculo afetivo naunidade de terapia intensiva neonatal: um desafio para as enfermeiras. Revista da Escola de Enfermagem da USP, 43(4), 49-55.

Costa, R. C., Klock, P. \& Locks, M. O. H. (2012). Acolhimento na unidade neonatal: percepção da equipe de enfermagem. Revista de Enfermagem da UERJ, 20(3), 349-53.

Ferreira, M. B., Monteiro, D. R. \& Souza, T. C. (2020). Em busca da humanização na UTI neonatal: método mãe canguru. Research, Society and Development. 9(11), e3089119631.

Hennig, M. A. S., et al. (2010). Atenção humanizada ao recém-nascido de baixo-peso. Método canguru e cuidado centrado na família: correspondências e especificidades. Physis Revista de Saúde Coletiva, Rio de Janeiro-RJ. 20(3). 835-52.

Lélis, A. L. P., Machado, M. F. A. S. \& Cardoso, M. V. L. M. L. (2009). Educação em saúde e a prática de enfermagem ao recém-nascido prematuro. Revista da Rede de Enfermagem do Nordeste-RENE, 10(4), 60-9.

Machado, A. C., Girão, G. C. M., Rodrigues, L. C. L., Silva, R. C. \& Rocha, S. S. (2012). Produção de conhecimento sobre o método canguru. Revista Interdisciplinar NOVAFAPI, 5(1), 61-7.

Maia, J. A., Oliveira, M. P., Furtado, S. S., Silva, L. M. \& Pereira, M. L. B. (2011). Método canguru: a importância da família na recuperação do recémnascidode baixo peso. Enfermagem em Foco, 2(4), 231-4. 
Research, Society and Development, v. 10, n. 10, e527101019280, 2021

(CC BY 4.0) | ISSN 2525-3409 | DOI: http://dx.doi.org/10.33448/rsd-v10i10.19280

Matos, E., Pires, D. E. P. \& Campos, G. W. S. (2009). Relações de trabalho em equipes interdisciplinares: contribuições para a constituição de novas formas de organização do trabalho em saúde. Revista Brasileira de Enfermagem, 62(6), 863-9.

Moreira, J. O., Romagnoli, R. C., Dias, D. A. S. \& Moreira, C. B. (2009). Programa mãe-canguru e a relação mãe-bebê na rede pública de Betim: pesquisa qualitativa. Psicologia em Estudo, 14(3), 475-83.

Neves, P. N., Revelli, A. P. X. \& Lemos, J. R. (2010). Atenção humanizada ao recém-nascido de baixo-peso método mãe canguru: percepções de puérperas. Revista Gaúcha de Enfermagem, 31(1), 48-54.

Queiroz, N. A. \& Maranhão, D. G. (2012). Ações e cuidados de enfermagem na implementação do método mãe-canguru. Revista de Enfermagem da UNISA, $13(2), 121-5$.

Sá, F. E., Sá, R. C., Pinheiro, L. M. \& Callou, F. E. (2010). Relações interpessoais entre os profissionais e as mães de 70 prematuros da unidadecanguru. Revista Brasileira em Promoção da Saúde, 23.(2), 144-9.

Santana, J. C. B., Assis, A. P. O., Silva, C. C. D. \& Quites, H. F. O. (2013). Método mãe canguru e suas implicações na assistência: percepção da equipede enfermagem. Enfermagem Revista, 16(1), 34-48.

Schmidt, K. T., Bessa, J. B., Rodrigues, B. C., Arenas, M. M., Corrêa, D. A. M. \& Higarashi, I. H. (2011). Recém-nascidos prematuros e a alta hospitalar: Uma revisão integrativa sobre a atuação da enfermagem. Revista da Rede de Enfermagem do Nordeste - RENE, 12(4), 849-58.

Silva, J. R., Thomé, C. R. \& Abreu, R. M. (2011). Método mãe canguru nos hospitais/maternidade públicos de Salvador e atuação dos profissionais da saúde na segunda etapa do método. Revista CEFAC, 13(3), 522-33.

Silva, H. L. L., et al. (2020). Percepção materna diante da utilização do método canguru: uma revisão integrativa. Research, Society and Development. 9(7), e886975146.

Silva, A. R. E., Garcia, P. N. \& Guarigilia, D. A. (2013).Método canguru e os benefícios para o recém-nascido. Revista Hórus, 7(2), 1-11.

Veras, R. M. \& Traverso-Yépez, M. A. (2011). O cotidiano institucional do método mãe canguru na perspectiva dos profissionais de saúde. Psicologia \& Sociedade, 23(1), 90-8. 